\title{
Multiplex ligation dependent probe amplification - A useful, fast and cost-effective method for identification of small supernumerary marker chromosome in children with developmental delay and congenital heart defect
}

\author{
George Andrei Crauciuc ${ }^{1}$, Florin Tripon ${ }^{1 *}$, Alina Boglişs ${ }^{1}$, Amalia Făgărăşan², \\ Claudia Bănescu ${ }^{1}$
}

1. Genetics Department, University of Medicine, Pharmacy, Sciences and Technology of Târgu Mureş, Romania, Romania Genetics Laboratory, Center for Advanced Medical and Pharmaceutical Research,

University of Medicine, Pharmacy, Sciences and Technology of Târgu Mureş, Romania

2. Department of Pediatrics III, Cardiology Clinic II - Children, University of Medicine, Pharmacy,

Sciences and Technology of Târgu Mureş, Romania

\begin{abstract}
Small supernumerary marker chromosome (SSMC) is a rare chromosomal abnormality and is detected in about $0.3 \%$ in cases with multiple congenital anomalies (MCA) and/or developmental delay. Different techniques for investigation of cases with MCA and/or developmental delay are available ranging from karyotyping to molecular cytogenetic technique and ultimately multiplex ligation dependent probe amplification (MLPA). Here we present a patient with multiple congenital anomalies for which classical cytogenetic technique was used as a first step in diagnosis and the results being confirmed by MLPA. The karyotype disclosed a SSMC considered to be a fragment of chromosome 22. The MLPA analysis using SALSA MLPA probemix P064-C2 Microdeletion Syndromes-1B confirmed the karyotype results, and according to the manufacturer's recommendation we performed another confirmation analysis with MLPA probemix P311-B1 Congenital Heart Disease and MLPA probemix P250-B2 DiGeorge. We also suspected an Emanuel syndrome and performed another MLPA analysis with SALSA MLPA probemix P036-E3 Subtelomeres Mix 1 and probemix P070-B3 Subtelomeres Mix 2B for investigation of subtelomeric region that revealed a duplication of $11 q 25$ region and the confirmation was performed using SALSA MLPA probemix P286-B2 Human Telomere-11.

In conclusion, we consider that MLPA is a valuable method for identification of SSMC in children with developmental delay and congenital anomalies. Genetic diagnosis using different molecular techniques, such as MLPA, for increasing accuracy in identification of chromosomal structural aberrations has an important role in clinical
\end{abstract}

* Corresponding author: Florin Tripon, University of Medicine, Pharmacy, Sciences and Technology of Târgu Mureş, Romania. E-mail: tripon.florin.2010@gmail.com 
diagnosis and in genetic counselling and our case explain the importance of using a specific laboratory technique for each stage of diagnosis.

Keywords: supernumerary marker chromosomes, multiplex ligation dependent probe amplification, congenital anomalies

Received: $6^{\text {th }}$ August 2018; Accepted: $18^{\text {th }}$ September 2018; Published: $3^{\text {rd }}$ October 2018

\section{Introduction}

Congenital heart disease (CHD) is the most frequent type of congenital malformation with a relative incidence of $6 / 1000$ live births and a small percentage of this CHD is related to chromosomal abnormality described in literature $(1,2)$. An important cause of mortality and morbidity is represented by the malformation itself and by the complications which may occur in the long term $(3,4)$. In the majority of these cases the etiology remains unknown, but among the known causes of congenital heart disease, approximately one third of cases is associated with genetic factors such as chromosomal anomalies identified in about $0.4-26.8 \%$ of all CHD, copy number variants (CNVs), gene mutations (5-8). Based on the findings reported by Pânzaru et al in a study that included 1123 children with multiple congenital anomalies of which 321 patients had CHD, the frequency of chromosomal aberrations varied by type of CHD and was higher in patients with atrioventricular canal (AVC), persistent ductus arteriosus (PDA), ventricular and atrial septal defects (VSD, ASD) (9).

Chromosomal aberrations consisting of CNVs, like microduplication or microdeletion, are described as a major cause in the etiology of CHD, with a mean value of $14 \%$ in syndromic $\mathrm{CNVs}$ and 7\% in non-syndromic CNVs $(5,10,11)$. In addition, Karen et al. classified the CNVs as follow: the first type are CNVs correlated with microdeletion syndromes, from which we can highlight those associated with CHD (like DiGeorge or William's syndromes) or due to chromosomal rearrangements, chromosomal mosaicism or partial aneuploidy; the second type are CNVs that contain genes involved in embryogenesis of the heart development (GATA4); and in the third category the CNVs described to be correlated with a variety of other phenotypes (12). Microdeletions/ microduplications in patients with intellectual disability syndrome; intellectual disability associated with dysmorphic features and/ or multiple congenital abnormalities may be detected by using fluorescence in situ hybridization (FISH) and Multiplex Ligation-dependent Probe Amplification (MLPA) (13).

Recently, the introduction and use of microarray technology has led to the identification of numerous syndromes with microdeletions and microduplications by submicroscopic detection of CNVs with unprecedented resolution, now in patients with intellectual disability and/ or multiple congenital anomalies, microarray test or whole exome sequencing may be considered as a first-tier diagnostic test (14). However, this new technology requires consumables that are hardly accessible to all diagnostic centers and of course, expensive equipment (15).

Despite modern cytogenetics and significant advances in molecular-genetic technologies such as FISH and comparative genomic hybridization (CGH), karyotyping and MLPA technique continue to be a crucial and basic tool in genetic evaluation, even for developing countries.

Here, we present the importance of classical cytogenetic methods in the diagnosis procedure in one case with multiple congenital anomalies and intellectual disability and the impact of MLPA technique for CNVs analysis in confirma- 
tion of chromosomal abnormalities. Secondly, we aimed to assess which region of CNVs gives certain phenotypical changes when the karyotyping methods cannot establish it exactly.

\section{Material and methods}

\section{Clinical report}

We investigated a patient referred to the Genetic department in January 2018, at the age of one and a half years old, born at term after an imminent abortion during the second semester of pregnancy with healthy parents (26-year-old mother and 27-year-old father) and unrelated. $\mathrm{He}$ is the first child in the family born by cesarean section delivery, with a birth weight of $2200 \mathrm{~g}$. After birth, the neonate was submitted to a surgical intervention for cleft lip and palate. He was diagnosed with an atrial septal defect accusing an effort intolerance and profuse sweating. Echocardiography revealed a large atrial septal defect (ASD) and scrotal ultrasound show bilateral high undescended testicles. Neurological evaluation highlighted a hypotonic syndrome with a retard in psychomotor development because he did not sit unsupported, but kept rolling front to back and was walking with support. Dysmorphological examination showed microcephaly, downslanting palpebral fissures, long lashes, thick eyebrows, protruding nose with wide base, long philtrum, downturned corners of the mouth and microretrognathia. Taking into account the patient's clinical phenotype, cytogenetic testing was recommended.

\section{Cytogenetic Analysis}

Cytogenetic analysis was performed form peripheral blood lymphocytes, cultures were stimulated with phytohemagglutinin and were carried out according to the standard protocol using air dropping technique. G-banding with standard staining at a 400-500 band level was performed according to ISCN 2013. The pa- tient karyotype disclosed a numerical aberration $47, \mathrm{XY},+$ mar and the small sSMC was considered to be a fragment of chromosome 22. Considering the clinical characteristics of our patient and karyotyping analysis we raised the suspicion of Emanuel syndrome. The karyotyping analysis was recommended for parents but to date they have postponed the investigation.

\section{Multiplex Ligation Dependent Probe Am- plification}

Following the cytogenetic results, genomic DNA was extracted from peripheral blood leukocytes using iPrep PureLink gDNA Blood Kit (Invitrogen, Carlsbad, CA, USA) in the iPrep purification instrument.

In addition to cytogenetic analyses, we performed MLPA for investigation of the most common copy number changes associated with microdeletion and microduplication syndromes that include intellectual disability, CHD, etc. The MLPA (MRC Holland, Amsterdam, Holland) analysis was performed, in the first step, using commercial kit for exons located at the chromosome 22, namely SALSA MLPA P064 Microdeletion Syndrome-1B probemix from MRC-Holland (Amsterdam, Netherlands) and all the abnormalities identified by this kit were confirmed using syndrome-specific MLPA kits. All the procedures (DNA denaturation, hybridization reaction, ligation reaction, PCR reaction, fragment separation by capillary electrophoresis) were performed according to the manufacturer's protocol.

Fragments were analyzed by capillary electrophoresis using the Applied Biosystems 3500 Genetic Analyzer with a $50 \mathrm{~cm}$ array using POP7 polymer. Result analyses were achieved using the Coffalyser.Net software. The number of copies for region of interest were calculated using final ratio; the normal corresponded value was between 0.7 and 1.3. 
Ethics committee approval for genetic testing was obtained from the Ethics Committee of the University of Medicine and Pharmacy of Tîrgu Mureș, Romania.

\section{Results}

The MLPA analysis using SALSA MLPA probemix P064-C2 Microdeletion Syndromes-1B revealed a large duplication for the region $22 \mathrm{q} 11.1-\mathrm{q} 11.23$, containing the genes: CLTCL1 exon 3, CDC45 exon 1 and DGCR8 exon 2 (Figure 1).

According to Product Description SALSA MLPA probemix P064-C2 Microdeletion Syndromes-1B the results must be confirmed by another independent technique. Considering the result of karyotyping that highlights the presence of sSMC derivative and other studies which use a combination of MLPA kits in detection of chromosomal abnormalities we performed another analysis using the kit as follows: SALSA
MLPA probemix P311-B1 Congenital Heart Disease and SALSA MLPA probemix P250-B2 DiGeorge $(11,16)$. The P311 probemix contains probe for the following genes GATA4, NKX25, TBX5, BMP4, CRELD1 involved in normal heart development, and this kit contains 3 additional probes for the $22 \mathrm{q} 11$ region, with different genes and exons comparing with SALSA MLPA probemix P064 except CDC45 exon 1 gene. The fragment analysis with SALSA MLPA probemix P311 kit identified a heterozygous duplication for genes located in 22q11.21 (CDC45 exon 1, $G P 1 B B$ exon 2 and $D G C R 8$ exon 14) (Figure 2).

MLPA probemix P250-B2 DiGeorge that include probes for $22 \mathrm{q} 11.2$ region for detection of CNVs deletion or duplication associated with a variety of disorders DiGeorge syndrome (DGS; MIM 188400), Velocardiofacial syndrome (VCFS; MIM 192430) and Cat Eye syndrome (CES; MIM 115470). MLPA P250-B2 analyze revealed an extensive duplication for genes located in 22q11.1-q11.23 (IL17RA exon

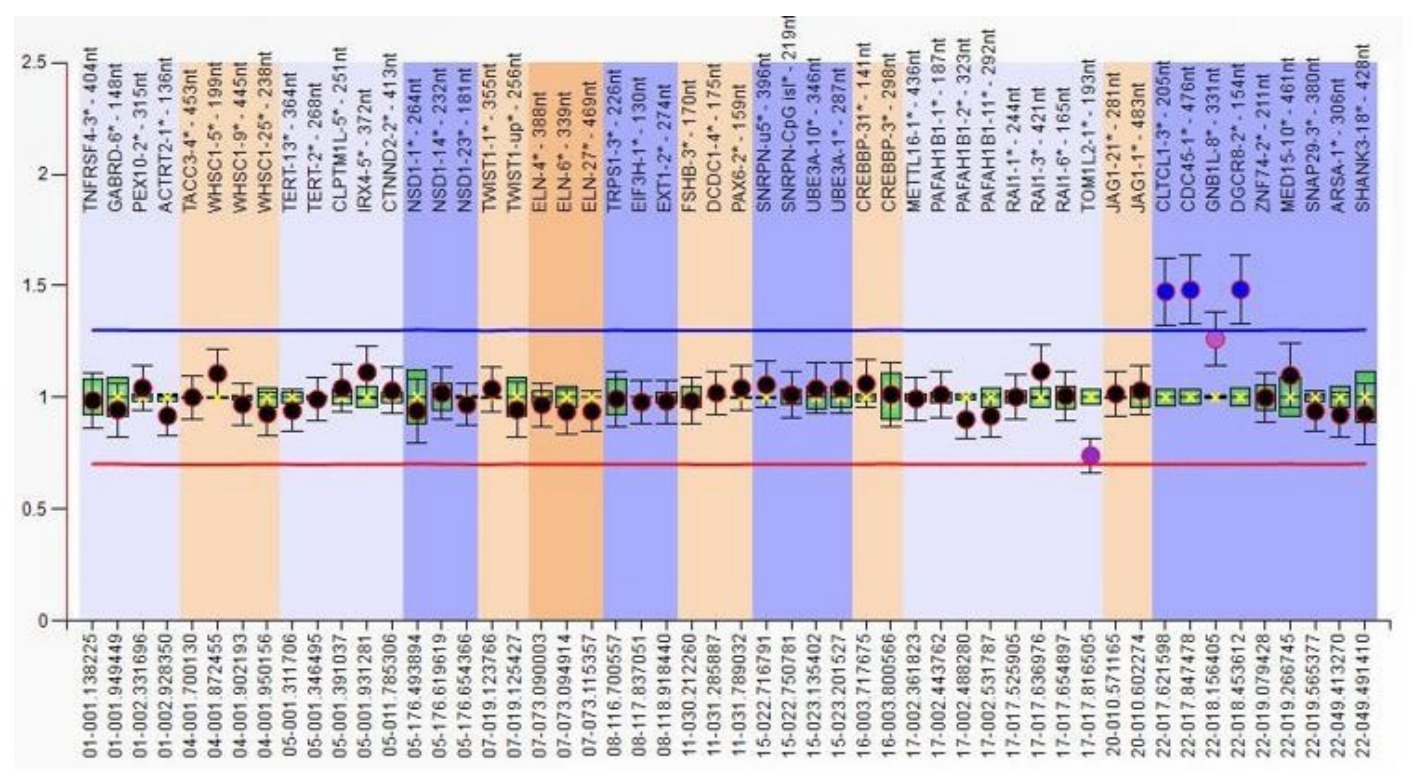

Fig. 1. Ratio chart for MLPA analysis using SALSA MLPA kit P064 Microdeletion Syndrome-1B probemix. Increase signal intensity (represented with blue bullets) corresponds to a heterozygous duplication 22q11.21. Duplication of this chromosomal segment involves the following genes: CLTCL1, CDC45, GNB1L, and DGCR8. 


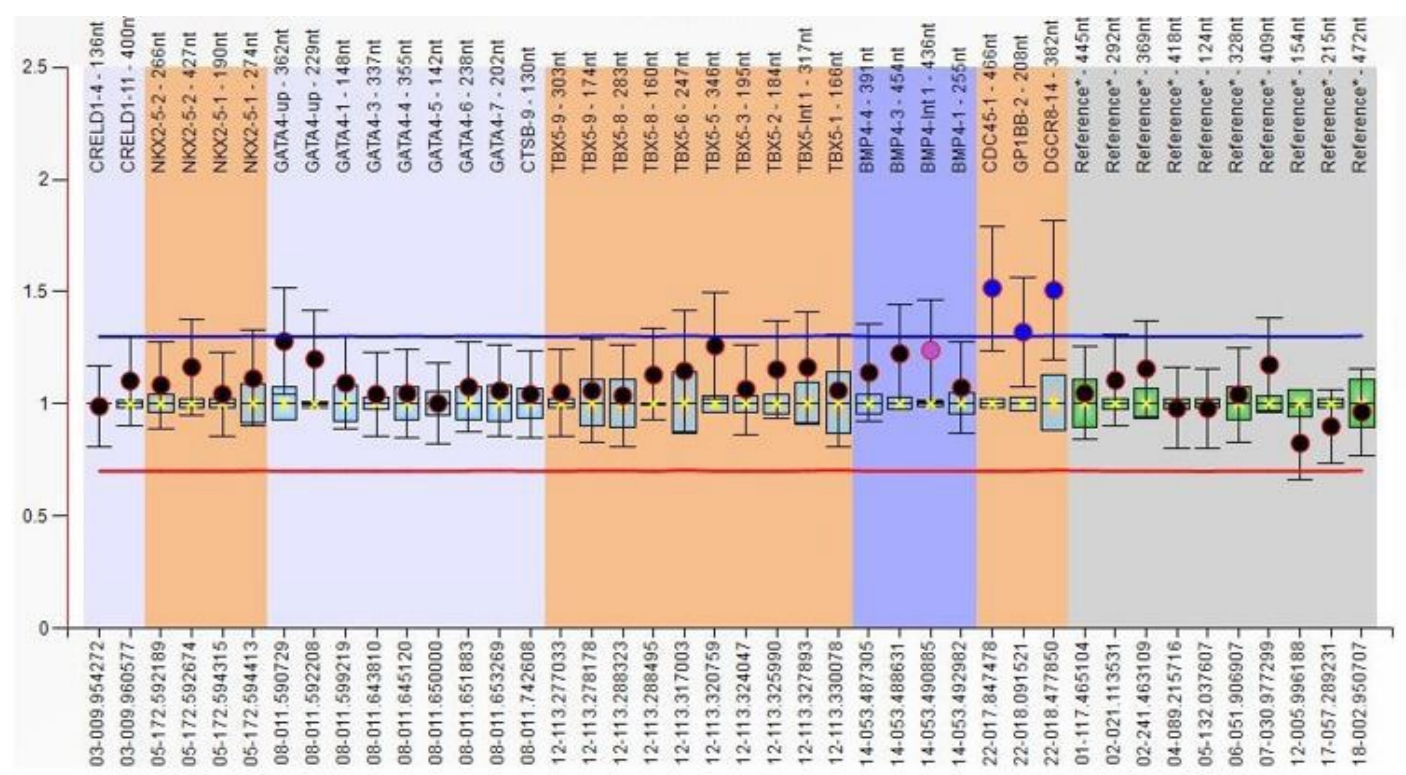

Fig. 2. Ratio chart for MLPA analysis using SALSA MLPA probemix P311-B1 Congenital Heart Disease indicate a heterozygous duplication of 22q11.21, in this duplication, represented with blue color bullets, are as follow $C D C 45, G P 1 B B$, and $D G C R 8$.

4, SLC25A18 exon 1, BID exon 4, MICAL3 exon 20, USP 18 exon 1, CLTCL1 exon 3, HIRA exon 25, CDC45 exon $1, C L D N 5$ exon $1, G P 1 B B$ exon 2, TBX1 exon 2, TBX1 exon 7, TXNRD2 exon 9, DGCR8 exon 2, ZNF74 exon 2, KLHL22 exon 2, MED15 exon 10, SNAP29 exon 5, LZTR1 exon 16, HIC2 exon 2, PPIL2 exon 20, TOP3B exon 7, RTDR1 exon 6, GNAZ exon 3, RTDR1 exon 2, RAB36 exon $1, S M A R C B 1$ exon 1 and SMARCB1 exon 9).

According to the phenotype, confirmation of 22q11.2 region duplication, confirmation by facial recognition by using Face-to-Gene (https://www.face2gene.com/) and description made by Zou et al. and Liehr the final diagnosis was Emanuel syndrome or the so-called "derivative chromosome 22 syndrome". Emanuel syndrome is frequently caused as a translocation between chromosome 22q11.2 and 11q23 present in one of the parents $(17,18)$. For complementary investigation, for subtelomeric 11q region we performed an additional MLPA anal- ysis using MLPA probemix P036-E3 Subtelomeres Mix 1 and P070 Subtelomeres Mix 2B considering the recommendation of the MRC Holland to use both MLPA probemix P036 and P070 for testing because all P070 probes are different from P036 kit.

MLPA probemix P036-E3 Subtelomeres Mix 1 content 2 probes for each subtelomeric region, except for acrocentric chromosomes $(13,14,15,21,22)$ are recommended for primary screening of subtelomeres. The results with increased signal intensity and final ratio $>$ 1.3 indicate a heterozygous duplication of gene NCAPD3 exon 2 located in 11.q25 region (Figure 3A). SALSA MLPA P070 Subtelomeres Mix 2B probemix that contains probes for $11 \mathrm{q} 25$ for gene $I G S F 9 B$ and also for $22 \mathrm{q} 11.1$ ILI $R R A$ exon 4, and we noticed a duplication in both implicated regions from $11 \mathrm{q} 25$ and 22q11.1 (Figure 3B).

CNVs detected by $\mathrm{P} 036$ or P070 must be verified by a designated MLPA follow-up probe- 
A

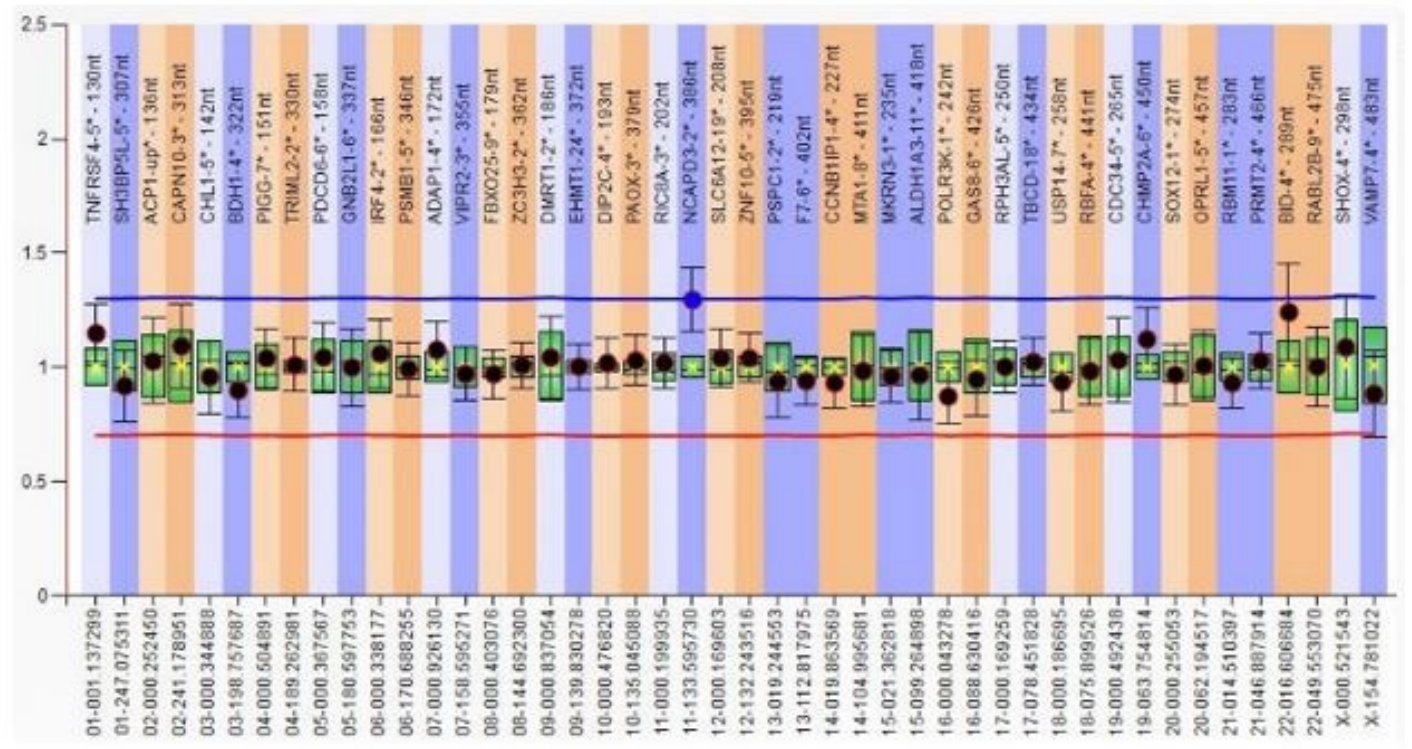

B

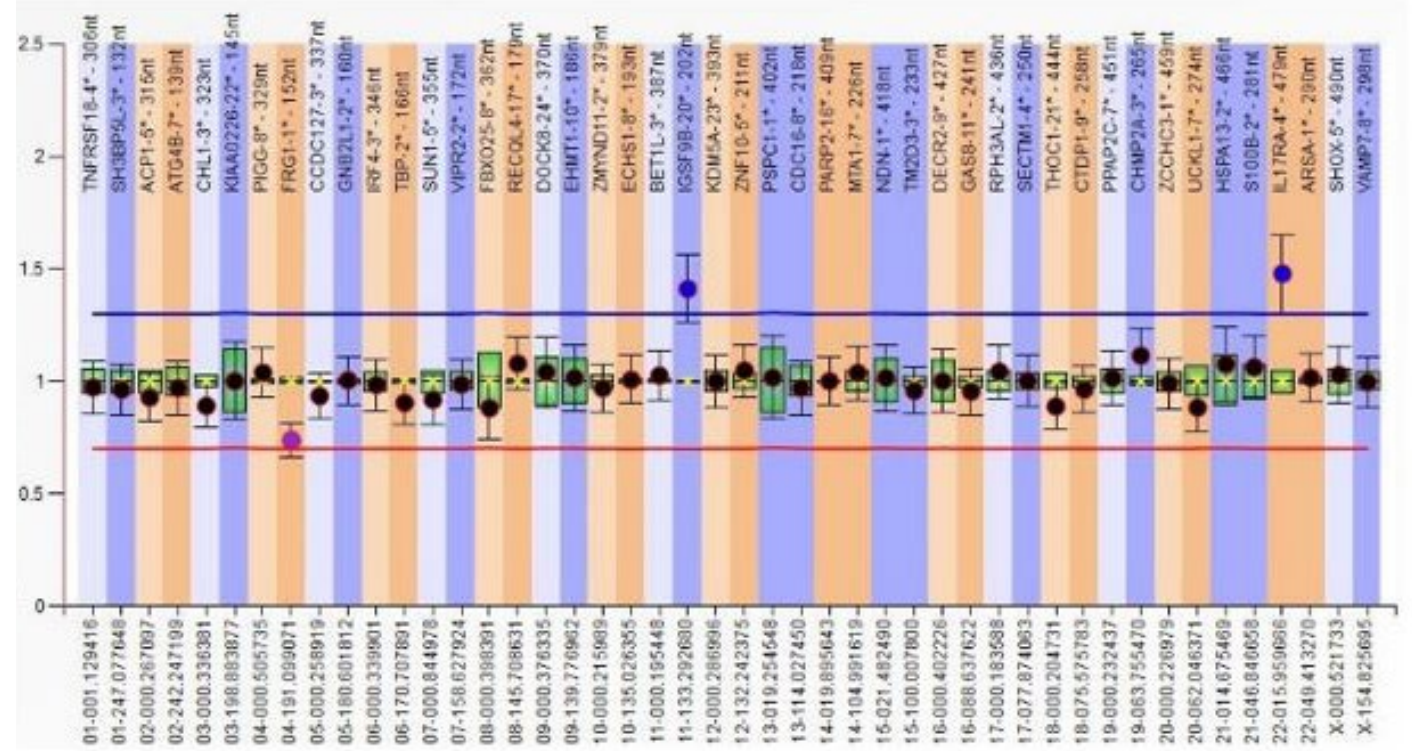

Fig. 3. Figure 3A Ratio chart for SALSA MLPA probemix P036-E3 Subtelomeres Mix signal intensity increase and final ratio $>1.3$ indicate a heterozygous duplication of gene NCAPD3 exon 2 located in 11.q25 region. Figure 3B Ratio chart for SALSA MLPA probemix P070 Subtelomeres Mix 2B indicate a heterozygous duplication for genes located in $11 \mathrm{q} 25$ and $22 \mathrm{q} 11.1$ region.

mix. In this regard, we used SALSA MLPA probemix P286-B2 Human Telomere-11 for confirmation of the results obtained with MLPA probemix P036-E3 Subtelomeres Mix 1.
Results for SALSA MLPA probemix P286-B2 confirmed a heterozygous duplication for 11q24.3-q25 for exons of the gene analyzed in this region, as follow: NFRKB exon 4, APLP2 
exon 18, NTM exon 6, OPCML exon 7, OPC$M L$ intr 1, SPATA19 exon 5, NCAPD3 exon 2, $B 3 G A T 1$ exon 2 (Figure 4).

\section{Discussion}

The first step, for primary identification in cytogenetic techniques is the karyotyping which remains a precise and feasible method especially in small supernumerary marker chromosome (sSMC) (19). sSMC are detected in about $0.3 \%$ of cases with multiple congenital anomalies (MCA) and/or developmental delay (20,21). Classical karyotyping detects abnormalities in $3-15 \%$ of cases with multiple congenital malformation (22). Often, using classical cytogenetics technique, the dimension of the sSMC does not allow accurate determination of the chromosome origin. The confirmation of the origin/ provenance of sSMC is mandatory for an accurate diagnosis. For the first step in diagnosis confirmation the whole-genome array (WGA) screening has been frequently recommended. The major disadvantage for WGA is linked to costs, followed by FISH method when it is compared with traditional karyotyping (22).

Thus, the MLPA is a viable alternative because it is a low-cost usable technique, which is a rapid and sensitive determination in molecular screening for sSMC through CNVs analysis. This technique allows for quantity evaluation of up to 50 amplicons in one single reaction using a small amount of the DNA or RNA sample. It was introduced in 2002 by MRC Holland and it is currently available with 400 panels kit with a related short time to results and high sensitivity and simplicity (https://mlpa.com).

In the literature, multiple studies describe the usefulness of using a combination of MLPA kits for determining the chromosomal abnormality with a range results between 3.2-33.33\% which depend on the kit that was used and the patient group selection $(11,23)$.

Moreover, MLPA is able to detect the gene dosage disruption and in our case, the gene identified in duplicated region was investigated us-

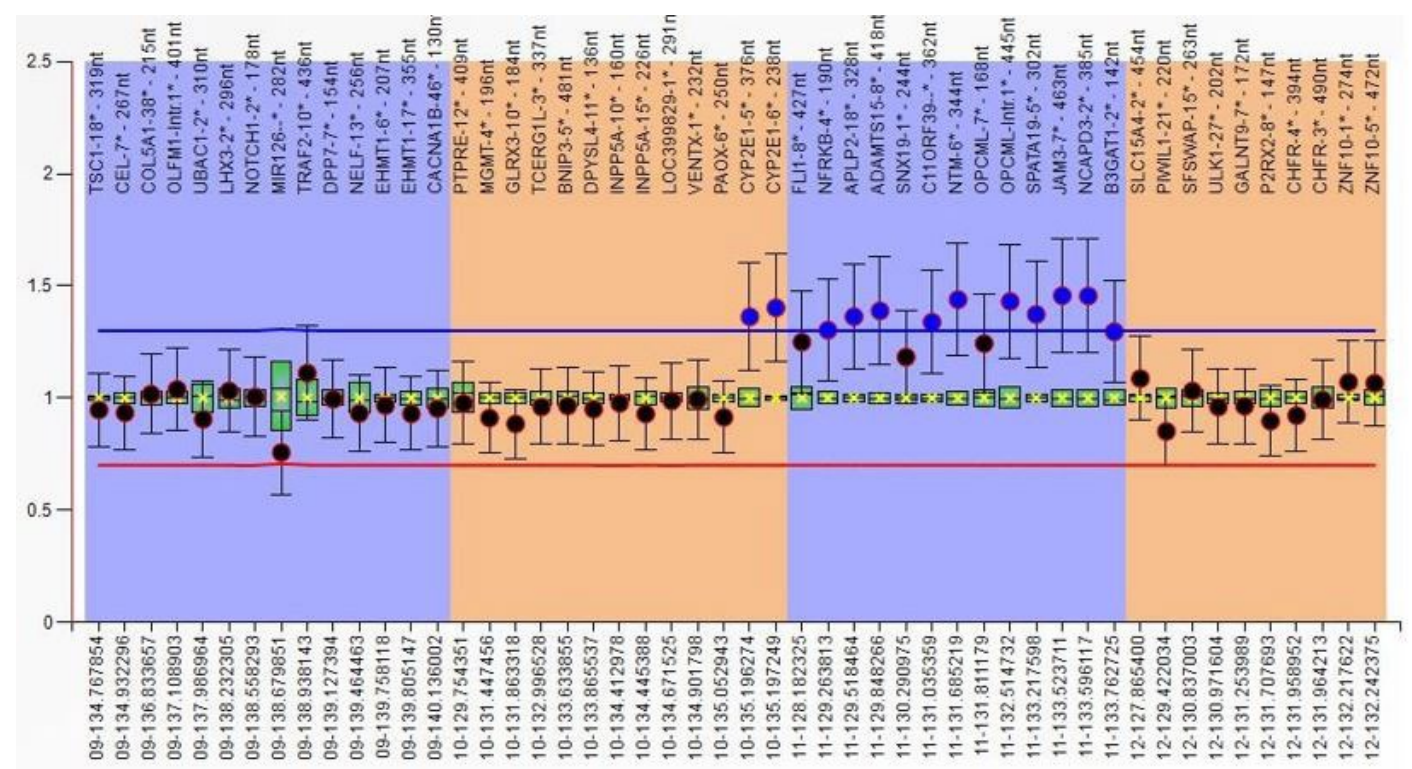

Fig. 4. Ratio chart for MLPA analysis using SALSA MLPA probemix P286-B2 Human Telomere-11 confirm a heterozygous duplication for chromosome 11 (11q24.3-q25 region). 
ing websites: https://decipher.sanger.ac.uk/ and http://omim.org. CNV duplication syndrome detected with MLPA P064 for genes CLTCL1, CDC45, GNB1L, and DGCR8, has been correlated in $97 \%$ cases with mental retardation and learning disability, in $67 \%$ with delayed psychomotor development, $63 \%$ growth retardation and $43 \%$ muscular hypotonia according with Wentzel et al. identically to CNVs duplication syndrome detected with P311 kit (24). The CNVs duplication syndrome and gene duplication detected with P250 kit are described as associated with abnormality of the nervous system, abnormality of the head or neck, and abnormality of limbs (https:// mlpa.com). NCAPD3 gene located in 11.q25 region is phenotypically associated with small size for gestational age, trigonocephaly, feeding difficulties in infancy, proportionate short stature, ptosis, renal hypoplasia.

In this case, the follow-up method for karyotypes which identified an SSMC was the MLPA technique, a successful method to establish the chromosomal fragments involved in the formation of sSMC. The genetic content of sSMC is involved directly in the phenotypic abnormalities. Multiple studies describe the use of WGA and FISH for sSMC but compared with these, the MLPA technique has a significant advantage such as one reaction can determine the chromosomal gene content for the unique-sequence positive sSMC, although targeted FISH is sometimes necessary to determine the structure of the sSMC and is often used following an abnormal array result (25).

Considering the investigated case and the many kits used, karyotyping remains the gold standard in setting microscopic chromosome changes. In order to confirm the results of the karyotype, MLPA is an advantage especially due to the possibility of detecting duplications and deletions of a single exon from a gene. Other advantages are about costs, by reducing them, short processing time, and increased specificity through the possibility of confirmation with other kits.

According to our clinical case, the utility of these techniques has been major. Firstly, by confirming the presence of a supernumerary chromosome marker derived from chromosome 22 by highlighting genes at this level, and secondly, the possibility of identifying the presence of a duplication of a region in chromosome 11, therefore MLPA analysis revealed a microduplication at $11 \mathrm{q} 25$ and $22 \mathrm{q} 11.1$. As such, the patient presents partial trisomy for genes located in $11 \mathrm{q} 25$ and $22 \mathrm{q}$ region. The duplicated genes revealed by MLPA analysis and previously published cases of Emanuel syndrome are phenotypically similar to our patient (2). In this case, the genetic techniques used confirm our clinical diagnosis and allow for a proper genetic counseling regarding the risks of recurrence.

We concluded that the association of CHD represented by ASD, developmental delay, hypotonic syndrome, dysmorphic features are caused by the microduplication of chromosome 11 and 22 , based on our CNVs analysis performed.

In conclusion, we consider that MLPA is a useful, fast and cost-effective method for identification of small supernumerary marker chromosome in children with developmental delay and congenital heart defect. Genetic diagnosis using different molecular techniques for increasing the accuracy in identification of chromosomal structural aberrations has an important role in the genetic counseling and our case explains the importance of using a specific laboratory technique for each stage of the diagnosis.

\section{Conflict of interest}

The authors declare no conflict of interest.

\section{Authors' contributions}

AC designed the study, collected the clinical data and wrote the manuscript and approved the 
final manuscript. FT performed DNA isolation, MLPA analysis and interpreted the results, read and approved the manuscript. AB performed cytogenetic analyses, and MLPA analysis and read and approved the manuscript. AF collected blood sample, performed ultrasound investigation, read and approved the manuscript. CB designed the study, interpreted the results, read the draft of the manuscript, revised it critically and approved the final version of manuscript.

\section{Abbreviations}

CHD - congenital heart disease

CNVs - copy number variants

AVC - atrioventricular canal

PDA - persistent ductus arteriosus

VSD - ventricular septal defects

ASD - atrial septal defects

FISH - fluorescence in situ hybridization

MLPA - multiplex ligation-dependent probe amplification

CGH - comparative genomic hybridization

WGA - whole-genome array

\section{References}

1. Osoegawa K, Iovannisci DM, Lin B, Parodi C, Schultz $\mathrm{K}$, Shaw GM, et al. Identification of Novel Candidate Gene Loci and Increased Sex Chromosome Aneuploidy among Infants with Conotruncal Heart Defects. Am J Med Genet A. 2014 Feb;0(2):397-406. DOI: 10.1002/ ajmg.a.36291

2. Bernstein D. Epidemiology and genetic basis of congenital heart disease. Kliegman R, Stanton B, St Geme JW, Schor NF, Behrman RE, Nelson Textbook of Pediatrics, Philadelphia, 2016, pps 2182-2187.

3. Murray LE, Smith AH, Flack EC, Crum K, Owen J, Kannankeril PJ. Genotypic and phenotypic predictors of complete heart block and recovery of conduction after surgical repair of congenital heart disease. Heart Rhythm. 2017 Mar;14(3):402-9. DOI: 10.1016/j. hrthm.2016.11.010

4. Muntean I, Şuteu C, Togănel R, Bănescu C. Association between MDR1 gene polymorphism and clinical course of pediatric pulmonary arterial hypertension. Rev Romana Med Lab. 2018;26(3):305-12. DOI: 10.2478/ rrlm-2018-0025

5. Cowan JR, Ware SM. Genetics and genetic test- ing in congenital heart disease. Clin Perinatol. 2015 Jun;42(2):373-93. DOI: 10.1016/j.clp.2015.02.009

6. Gelb BD, Chung WK. Complex Genetics and the Etiology of Human Congenital Heart Disease. Cold Spring Harb Perspect Med. 2014 Jul;4:a013953. DOI: 10.1101/cshperspect.a013953

7. Soares G, Alvares S, Rocha C, Teixeira MF, Mota $\mathrm{MC}$, Reis MI, et al. Congenital heart defects and chromosomal anomalies including 22q11 microdeletion (CATCH 22). Rev Port Cardiol. 2005 Mar;24(3):34971.

8. Rachel S, Ian JW, Jamie B, Rebecca D, Ana T, Diana Z, et al. Contribution of Global Rare Copy-Number Variants to the Risk of Sporadic Congenital Heart Disease. Am J Hum Genet. 2012 September;91(3):489-501. DOI: 10.1016/j.ajhg.2012.08.003

9. Pânzaru M, Rusu C, Voloşciuc M, Braha E, Butnariu L, Gramescu M, et al. Benefits of cytogenetic testing in diagnosis of plurimalformative syndromes with congenital heart defects. Rev Romana Med Lab. 2012;20(3):265-72.

10. Monteiro RAC, Freitas ML, Vianna GS, de Oliveira VT, Pietra RX, Ferreira LCA, et al. Major Contribution of Genomic Copy Number Variation in Syndromic Congenital Heart Disease: The Use of MLPA as the First Genetic Test. Mol Syndromol. 2017 Aug;8:22735. DOI: $10.1159 / 000477226$

11. Lander J, Ware SM. Copy Number Variation in Congenital Heart Defects. Curr Genet Med Rep. 2014;2(3):168-78. DOI: 10.1007/s40142-014-0049-3

12. Karen RS, Rafaella M, Janaina H, Lucia CP, Mariluce R. Cytogenomic Evaluation of Subjects with Syndromic and Nonsyndromic Conotruncal Heart Defects. Biomed Res Int. 2015; 2015:401941.

13. Schouten JP, McElgunn CJ, Waaijer R, Zwijnenburg D, Diepvens F, Pals G. Relative quantification of 40 nucleic acid sequences by multiplex ligation-dependent probe amplification. Nucleic Acids Res. 2002 Jun;30(12):e57. DOI: 10.1093/nar/gnf056

14. Slavotinek AM. Novel microdeletion syndromes detected by chromosome microarrays. Hum Genet. 2008 Aug;124(1):1-17. DOI: 10.1007/s00439-008-0513-9

15. Sireteanu A, Popescu R, Braha EE, Bujoran C, Butnariu L, Caba L, et al. Detection of chromosomal imbalances using combined MLPA kits in patients with syndromic intellectual disability. Rev Romana Med Lab. 2014;22(2):157-64. DOI: 10.2478/rrlm-2014-0019

16. Lam AC, Lam ST, Lai KK, Tong TM, Chau TC. High rate of detection of subtelomeric aberration by using combined MLPA and subtelomeric FISH approach in patients with moderate to severe mental retardation. Clin Biochem. 2006 Feb;39(3):196-202. DOI: 10.1016/j.clinbiochem.2006.01.003

17. Zou PS, Li HZ, Chen LS, Ma M, Chen XH, Xue D, et al. A rare case of trisomy 11q23.3-11q25 and tri- 
somy 22q11.1-22q11.21. Genetic and Molecular Research. 2016;15(2):gmr15028140. DOI: 10.4238/ gmr. 15028140

18. Liehr T, Chapter 5 Small Supernumerary Marker Chromosomes known to be correlated with specific syndromes, Small Supernumerary Marker Chromosomes (sSMC), Springer-Verlag Berlin Heidelberg, 2012, pp 47-52. DOI: 10.1007/978-3-642-20766-2 5

19. Trifonov V, Fluri S, Binkert F, Nandini A, Anderson J, Rodriguez L, et al. Complex rearranged small supernumerary marker chromosomes (sSMC), three new cases; evidence for an underestimated entity?. Mol Cytogenet. 2008 Apr;1:6. DOI: 10.1186/1755-8166-1-6

20. Sadek AA, Ashry MM. Yield of karyotyping in children with developmental delay and/or dysmorphic features in Sohag University Hospital, Upper Egypt. Egyptian J Med Hum Genet. 2018;19(3):253-9. DOI: 10.1016/j. ejmhg.2017.12.007

21. Hochstenbach R, Nowakowska B, Volleth M, Ummels A, Kutkowska-Kaźmierczak A, Obersztyn E, et al. Multiple Small Supernumerary Marker Chromosomes Resulting from Maternal Meiosis I or II Errors. Mol Syndromol. 2016 Feb;5(6):210-21.
22. Jehee FS, Takamori JT, Vasconcelos MPF, Pordeus AC, Latini FR, Bertola DR, et al. Using a combination of MLPA kits to detect chromosomal imbalances in patients with multiple congenital anomalies and mental retardation is a valuable choice for developing countries. Eur J Med Genet. 2011;54:425-32. DOI: 10.1016/j.ejmg.2011.03.007

23. Sørensen KM, El-Segaier M, Fernlund E, Errami A, Bouvagnet $\mathrm{P}$, Nehme N, et al. Screening of congenital heart disease patients using multiplex ligation-dependent probe amplification: Early diagnosis of syndromic patients. Am J Med Genet A. 2012 Apr;158A(4):720-5. DOI: 10.1002/ajmg.a.35214

24. Wentzel C, Fernström M, Ohrner Y, Annerén G, Thuresson AC. Clinical variability of the $22 \mathrm{q} 11.2$ duplication syndrome. Eur J Med Genet. 2008;51(6):501-10. DOI: 10.1016/j.ejmg.2008.07.005

25. Woori J, Hyojin C, Jiyeon K, Jung-Ok S, Seok CK, Bo Kyung K, et al. Identification of small marker chromosomes using microarray comparative genomic hybridization and multicolor fluorescent in situ hybridization, Mol Cytogenet. 2016;9:61. DOI: 10.1186/s13039-0160273-5 